\title{
UNIQUENESS THEOREMS FOR CERTAIN IMPROPERLY POSED PROBLEMS ${ }^{1}$
}

\author{
BY EUTIQUIO C. YOUNG
}

Communicated by Philip Hartman, August 31, 1970

1. Introduction. It is well known that the Dirichlet problem for hyperbolic equations is in general an improperly posed problem. In the case of the two dimensional wave equation $u_{t t}-u_{x x}=0$, for instance, Bourgin and Duffin [1] have shown that in a rectangle with sides parallel to the coordinate axes uniqueness of solution of the Dirichlet problem holds if and only if the ratio of the sides of the rectangle is an irrational number. Recently, Dunninger and Zachmanoglou have extended Bourgin and Duffin's result to the $n$ dimensional wave equation [2] and to more general hyperbolic equations in cylindrical domains [3]. Corresponding result on the Neumann problem for the $n$-dimensional wave equation has also been given by Sigillito [4]. Extension of some of these results to singular equations have been carried out recently by Dunninger and Weinacht [5].

The purpose of this note is to announce some results on the uniqueness of solutions of the Dirichlet and Neumann problems for the singular hyperbolic equation

$$
L u \equiv u_{t t}+\frac{k}{t} u_{t}-\left(a^{i j} u_{x_{i}}\right)_{x_{j}}+c u=0
$$

where the coefficients $a^{i j}$ and $c$ are functions of the variables $x_{1}, \cdots$, $x_{n}$ alone and $k$ is a real parameter, $-\infty<k<\infty$. Here the repeated indices are to be summed from 1 to $n$. Conditions for uniqueness valid for various ranges of $k$ will be given. These results naturally contain all previous results obtained in [1]-[4].

Let $D$ be a bounded domain in $x=\left(x_{1}, \cdots, x_{n}\right)$ space and let $Q=D \times I$ where $I$ is the interval $0<t<T$. It is assumed that the coefficient matrix $\left(a^{i j}\right)$ is symmetric and positive definite and that $c \geqq 0$ in $D$. Moreover, it will be assumed that $a^{i j}, c$, and the boundary $\partial D$ of $D$ are sufficiently smooth in order to ensure the existence of a complete set of eigenfunctions for the eigenvalue problems that arise in the sequel.

AMS 1969 subject classifications. Primary 3501, 3553, 3555, 3576.

Key words and phrases. Improperly posed problems, uniqueness of solutions of the Dirichlet and Neumann problems, eigenvalues, eigenfunctions.

1 This work was supported by NSF grant GP-11543. 
2. The Dirichlet problem. Consider the homogeneous Dirichlet problem

$$
L u=0 \quad \text { in } Q, \quad u=0 \quad \text { on } \partial Q .
$$

We shall prove uniqueness of solution of this problem by showing that every smooth solution vanishes identically in $Q$. In the case when $k>0$ however, we see that every smooth solution vanishes identically in $\bar{Q}$ whenever it vanishes at $t=0$ and on the lateral surface of the cylinder $Q$.

THEOREM 1. Let $k>0$. If $u \in C^{2}(Q) \cap C^{1}(\bar{Q})$ is a solution of $L u=0$ such that $u=0$ at $t=0$ and on $\partial D$ for $0 \leqq t<T$, then $u \equiv 0$ in $\bar{Q}$ for any value of $T$.

The proof of this theorem hinges on the fact that every smooth solution of equation (1) satisfies the condition $u_{t}(x, 0)=0$ for any nonzero $k$. This fact was actually established in [6] in the special case when $\left(a^{i j}\right)$ is the identity matrix. However, the method employed there can be carried out here almost step for step to establish the same result for the more general equation (1) using the cylinder $Q$.

Now, to prove the theorem, we integrate the differential identity

$$
2 u_{t} L u=\left(u_{t}^{2}+a^{i j} u_{x_{i}} u_{x_{j}}-c u^{2}\right)_{t}-2\left(a^{i j} u_{x_{i}} u_{t}\right)_{x_{j}}+\frac{2 k}{t} u_{t}^{2}
$$

over the domain $Q_{s}=Q \cap\{0<t<s\}, s \leqq T$, and use the divergence theorem to obtain

$$
\begin{array}{r}
\int_{\partial_{Q_{s}}}\left[\left(u_{t}^{2}+a^{i j} u_{x_{i}} u_{x_{j}}\right.\right. \\
\left.\left.+c u^{2}\right) \nu_{t}-2 a^{i j} u_{x_{i}} u_{t} \nu_{j}\right] d S \\
+2 k \int_{Q_{s}} \int \frac{u^{2}}{t} d x d t=0 .
\end{array}
$$

Here $\left(\nu_{1}, \cdots, \nu_{n}, \nu_{t}\right)$ denotes the outward unit vector normal to $\partial Q_{s}$. By the boundary conditions satisfied by $u$ and the fact that $u_{t}(x, 0)=0$, equation $(4)$ reduces to

$$
\left.\int_{D}\left(u_{t}^{2}+a^{i j} u_{x_{i}} u_{x_{j}}+c u^{2}\right)\right|_{t=8} d x+2 k \int_{Q_{s}} \int \frac{u_{t}^{2}}{t} d x d t=0 .
$$

Since $\left(a^{i j}\right)$ is positive definite, $c \geqq 0$, and $k>0$, each term in (5) must be zero. Thus

$$
\left.\int_{D}\left(u_{t}^{2}+a^{i j} u_{x_{i}} u_{x_{j}}+c u^{2}\right)\right|_{t=s} d x=0
$$


for arbitrary value of $s, 0 \leqq s \leqq T$. This implies that $u$ is a constant in $Q$. Since $u=0$ initially (at $t=0$ ), it follows that $u \equiv 0$ in $\bar{Q}$ for any value of $T$.

On the other hand, when $k \leqq 0$ uniqueness holds if and only if the height $T$ of the cylinder satisfies certain condition. We have

THEOREM 2. If $k \leqq 0$, then every solution $u \in C^{2}(Q) \cap C^{1}(\bar{Q})$ of the problem (2) vanishes identically in $Q$ if and only if

$$
J_{(1-k) / 2}\left(\lambda_{m}^{1 / 2} T\right) \neq 0
$$

where $\lambda_{m}(m=1,2, \cdots)$ are the nonzero eigenvalues of the problem

$$
\begin{gathered}
\left(a^{i j_{v_{i}}}\right)_{x_{j}}-c v+\lambda v=0 \text { in } D, \\
v=0 \text { on } \partial D .
\end{gathered}
$$

The necessity proof of the theorem follows easily for if (7) is not satisfied, then

$$
J_{(1-k) / 2}\left(\lambda_{p}^{1 / 2} T\right)=0
$$

for some positive integer $p$. But then the function

$$
w(x, t)=t^{(1-k) / 2} J_{(1-k) / 2}\left(\lambda_{p}^{1 / 2} t\right) v_{p}(x)
$$

where $v_{p}$ is the eigenfunction corresponding to $\lambda_{p}$, is a nontrivial solution of the problem (2) as is readily verified.

The sufficiency proof which makes use of the completeness of the set of eigenfunctions of (8) is somewhat involved and is given in [7].

3. The Neumann problem. In the case of the homogeneous Neumann problem

$$
L u=0 \quad \text { in } Q, \quad \frac{\partial u}{\partial n}=0 \quad \text { on } \partial Q,
$$

where on the lateral surface of $Q$ the derivative $\partial u / \partial n$ is defined by

$$
\frac{\partial u}{\partial n}=a^{i j} u_{x_{i}} \nu_{j}
$$

$\left(\nu_{1}, \cdots, \nu_{n}\right)$ being the outward unit vector normal to $\partial D$, we have the following theorem.

THeOREM 3. If $u \in C^{2}(Q) \cap C^{1}(\bar{Q})$ is a solution of the problem (9), then $u \equiv 0$ (or $u=$ const. if $c=0$ ) for $k \geqq 0$ if and only if

$$
J_{(1+k) / 2}\left(\lambda_{m}^{1 / 2} T\right) \neq 0
$$


where $\lambda_{m}(m=1,2, \cdots)$ are the nonzero eigenvalues of the problem

$$
\begin{gathered}
\left(a^{i j_{x_{i}}}\right)_{x_{j}}-c v+\lambda v=0 \text { in } D, \\
\frac{\partial v}{\partial n}=0 \text { on } \partial D .
\end{gathered}
$$

The necessity of condition (10) actually holds for all values of $k$ and follows easily as in the case of Theorem 2. For if $\lambda_{p}$ is a nonzero eigenvalue of the problem (11) such that

$$
J_{(1+k) / 2}\left(\lambda_{p}^{1 / 2} T\right)=0
$$

then the function

$$
w(x, t)=t^{(1-k) / 2} J_{(k-1) / 2}\left(\lambda_{p}^{1 / 2} t\right) v_{p}(x)
$$

constitutes a nontrivial solution of the problem (9) for any value of $k$. In fact, it is easily shown that $L w=0$ and $\partial w / \partial n=0$ on $\partial D$ for $0 \leqq t$ $\leqq T$. Moreover, since

$$
\frac{\partial w}{\partial t}=-\lambda_{p}^{1 / 2} t^{(1-k) / 2} J_{(1+k) / 2}\left(\lambda_{p}^{1 / 2} t\right) v_{p}(x)=O(t)
$$

it follows that $w_{t}(x, 0)=0$ and, by $(12), w_{t}(x, T)=0$. Thus $\partial w / \partial n$ vanishes on $\partial Q$.

That condition (10) is also sufficient can be proved for $k \geqq 0$. The proof makes use of a technique that is different from that used for Theorem 2 and is contained in [7].

\section{REFERENCES}

1. D. G. Bourgin and R. Duffin, The Dirichlet problem for the vibrating string equation, Bull. Amer. Math. Soc. 45 (1939), 851-858. MR 1, 120.

2. D. R. Dunninger and E. C. Zachmanoglou, The condition for uniqueness of solutions of the Dirichlet problem for the wave equation in coordinate rectangles, J. Math. Anal. Appl. 20 (1967), 17-21. MR 37 \#1807.

3. - The condition for uniqueness of the Dirichlet problem for hyperbolic equations in cylindrical domains, J. Math. Mech. 18 (1969), 763-766. MR 38 \#4817.

4. V. G. Sigillito, On the uniqueness of solutions of certain improperly posed problems, Proc. Amer. Math. Soc. 24 (1970), 828-831.

5. D. R. Dunninger and R. J. Weinacht, Improperly posed problems for singular equations of the fourth order, Appl. Anal. (to appear).

6. D. W. Fox, The solution and Huygens' principle for a singular Cauchy problem, J. Math. Mech. 8 (1959), 197-220. MR 21 \#762.

7. E. C. Young, Uniqueness of solutions of the Dirichlet and Neumann problems for hyperbolic equations, Trans. Amer. Math. Soc. (to appear).

Florida State University, Tallahassee, Florida 32306 